\title{
Search for neutrinoless double beta decay with Gerda Phase II
}

\author{
K. von Sturm* on behalf of the GERDA Collaboration \\ INFN Sezione di Padova \\ Università degli Studi di Padova \\ E-mail: vonsturmepd.infn.it
}

The GERDA Collaboration is searching for neutrinoless double beta $(0 v \beta \beta)$ decay of the candidate isotope ${ }^{76} \mathrm{Ge}$ operating bare germanium detectors enriched in ${ }^{76} \mathrm{Ge}$ to about $88 \%$ in liquid argon (LAr). With the latest data release in May 2018 the best median sensitivity in the field of $T_{1 / 2}^{\beta \beta}>1.1 \cdot 10^{26} \mathrm{yr}$ has been reached. This was possible due to the ultra-low background level of $B I=\left(5.6_{-2.4}^{+3.4}\right) \cdot 10^{-4} \mathrm{cts} /(\mathrm{kg} \mathrm{keV} \mathrm{yr})$ which has been achieved thanks to an active veto system detecting LAr scintillation light, an improved granularity of the detector array improving anticoincidence background rejection, an extremely good energy resolution of $\mathrm{O}(0.1 \%)$ in the region of interest and an improved background rejection based on the distinct time profile with respect to signal events. A total exposure of $82.4 \mathrm{~kg} \mathrm{yr}$ accumulated in two experimental phases were analyzed an no evidence for $0 v \beta \beta$ decay was found. The obtained frequentist half-life limit is $T_{1 / 2}^{\beta \beta}>0.9 \cdot 10^{26} \mathrm{yr}$ at $90 \%$ C.L.

XIV International Conference on Heavy Quarks and Leptons (HQL2018)

May 27- June 1, 2018

Yamagata Terrsa, Yamagata,Japan

${ }^{*}$ Speaker. 


\section{Introduction}

In a double beta $(2 v \beta \beta)$ decay process two neutrons in the same nucleus are simultaneously transformed into two protons emitting two electrons and two anti-electron neutrinos. This decay is observed in nuclei in which the normal beta decay mode is energetically forbidden but a preferred energy level exists. If the neutrino is its own anti-particle, e.g. a Majorana particle, a lepton number violating decay mode, without the neutrinos in the final state, exists. Such process is beyond the standard model of particle physics and could explain the extremely small neutrino masses and the matter dominance over anti-matter in the universe. $2 v \beta \beta$ decay has been observed in about 10 isotopes, each of which is a candidate for the above described lepton number violating mode called neutrinoless double beta $(0 v \beta \beta)$ decay. The GERDA experiment [1] is designed to search for $0 v \beta \beta$ decay of the candidate isotope ${ }^{76} \mathrm{Ge}$. Its signature is the emission of two electrons with summed energy equal to the full energy ( $Q$-value) of the decay $Q_{\beta \beta}=2039.061 \pm 0.007 \mathrm{keV}$, resulting in a spectral line structure at $Q_{\beta \beta}$. In the following the experimental setup and recent results will be presented.

\section{The GERDA concept and Phase II setup}

The experimental setup of GERDA is located at the Laboratori Nazionali del Gran Sasso (LNGS) in Italy with an overburden of 3500 m.w.e. High-purity germanium (HPGe) detectors, enriched in ${ }^{76} \mathrm{Ge}$ to about $88 \%$, are operated bare in $64 \mathrm{~m}^{3}$ of liquid argon (LAr). They simultaneously serve as source and detector maximizing detection efficiency and have an exceptional energy resolution at $Q_{\beta \beta}$ of $O(0.1 \%)$. Their intrinsic impurity concentration is very low and their intrinsic background is thus negligible [2]. The surrounding stainless-steel cryostat is copper lined on the inside to shield the detectors from natural radioactivity. It is contained in a water tank which forms a passive shield and neutron moderator. Instrumented with 66 photo-multipliers (PMTs) of 8" diameter, the water tank also operates as an active Cherenkov muon veto [3]. A sketch of the full setup is shown in Fig. 1a).

For GERDA Phase II the experimental setup has been upgraded and improved [4]. The active detector mass is increased to $35.6 \mathrm{~kg}$ adding $20 \mathrm{~kg}$ of HPGe detectors of broad energy germanium (BEGe) $[5,6]$ type, which exhibit a superior energy resolution and pulse shape discrimination (PSD) potential. The detector array is mounted in a compact seven-string geometry, shown in Fig. 1c), in order to improve its granularity. This allows to more efficiently reject events depositing energy in multiple detectors via anti-coincidence. Each string is enclosed in a Tetra-Phenyl Butadiene (TPB) coated nylon mini-shroud creating a physical barrier - especially for ${ }^{42} \mathrm{~K}$, daughter of ${ }^{42} \mathrm{Ar}$, which is naturally present in LAr - but being translucent at the same time [7]. ${ }^{42} \mathrm{~K}$ decays via $\beta^{-}$decay to ${ }^{42} \mathrm{Ca}$ with an end-point energy of $\sim 3525 \mathrm{keV}$. The mini-shrouds limit the collection volume of this important background component. Parts of the setup are replaced by materials which are more radio-pure and components of an even more sparse design in order to keep natural radioactivity close to the detector-array at a minimum. The spring-loaded contact pins were replaced by aluminum wire bonds and the detectors are held by low-mass silicon holders (see Fig. 1d)). Furthermore, a hybrid LAr veto was mounted around the detector array consisting of light-guiding and TPB coated fibers coupled to silicon photo-multipliers (SiPMs)[8] and two arrays of low- 
a)

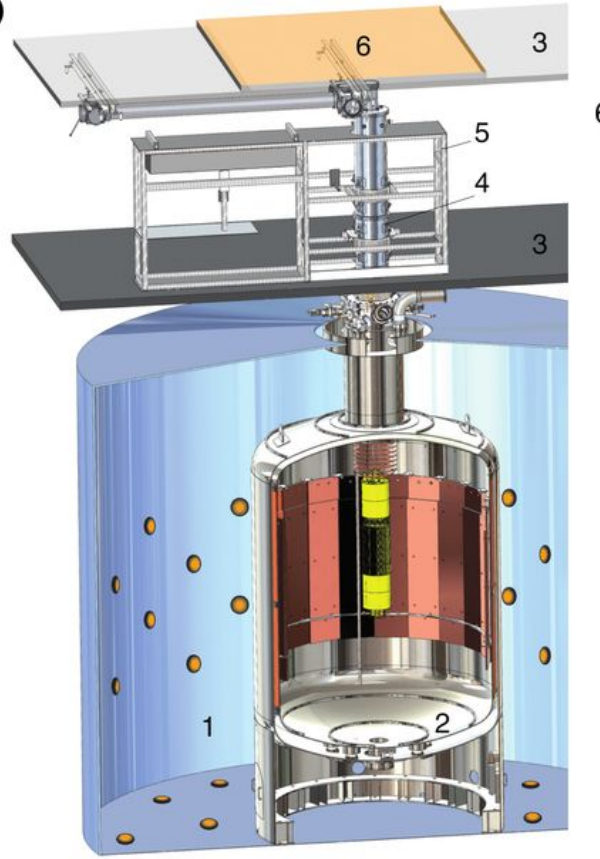

b)

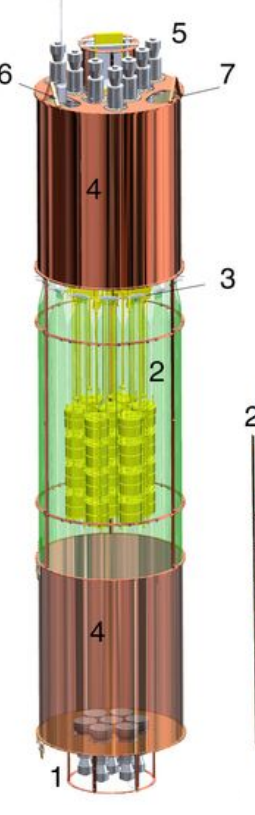

c)

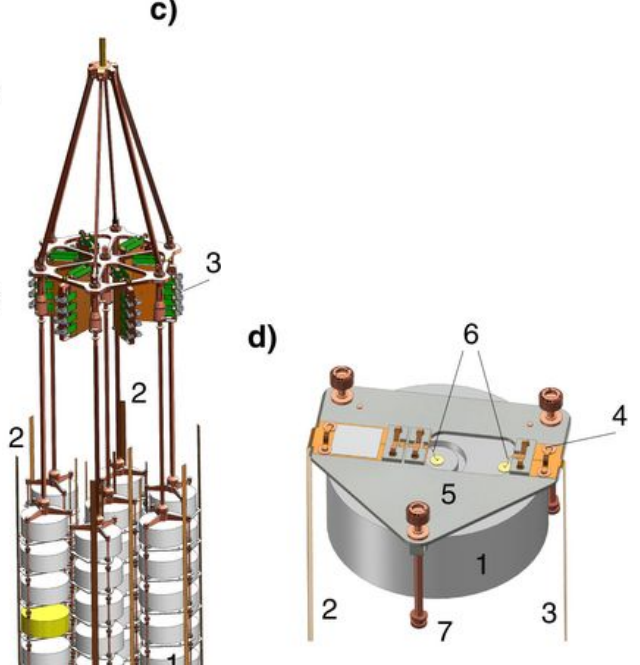

Figure 1: Schematic view of the GERDA Phase II experimental setup a) overview b) LAr veto system c) detector array d) detector module. Image taken from [10].

radioactivity 3-inch PMTs (see Fig. 1b)). It utilizes the scintillation light of the LAr in order to veto events depositing energy inside the cryo liquid.

Due to the short range of electrons in germanium signal-like events $2 v \beta \beta$ and $0 v \beta \beta$ events appear as point-like or single-site events (SSE) in a GERDA detector. In addition to passive shielding and the selection of radio-pure construction material, events with topologies that differ from SSE can be actively discarded. The goal is to suppress background to the limit of zero background in which no event in the region of interest (ROI: $Q_{\beta} \pm 5 \mathrm{keV}$ ) is expected for the full GERDA target exposure of $100 \mathrm{~kg}$ yr. The detector anti-coincidence and the LAr veto are methods of active background rejection. They are complemented by PSD which is the ability to distinguish events with multiple energy depositions and detector surface events in one detector from signal-like events. This is possible by analyzing the distinct time profile of different event types in the GERDA detectors [9].

\section{Performance and results}

GERDA adopts a staged approach, in a first experimental phase starting in November 2011 and lasting until September $201323.5 \mathrm{~kg}$ yr of physics data were collected with a background index of $B I=(1.1 \pm 0.2) \cdot 10^{-2} \mathrm{cts} /(\mathrm{kg} \mathrm{keV} \mathrm{yr})$ (Golden data set in [11]).

The ongoing Phase II data taking started in December 2015 with a target background level of $\mathrm{BI} \leq 10^{-3} \mathrm{cts} /(\mathrm{kg} \mathrm{keV} \mathrm{yr})$. Until April 2018 GERDA accumulated $58.9 \mathrm{~kg}$ yr of physics exposure in Phase II configuration. This divides into $28.1 \mathrm{~kg}$ yr from semi-coaxial detectors and $30.8 \mathrm{~kg} \mathrm{yr}$ from BEGe detectors. The exposure build-up and the livetime fraction over the full Phase II data 


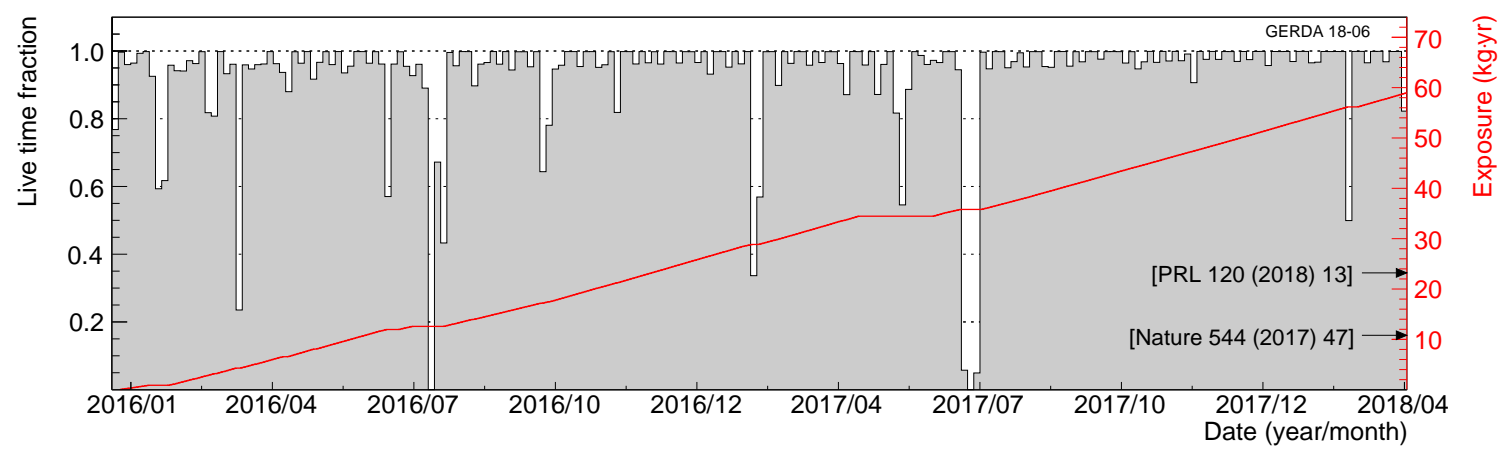

Figure 2: GERDA Phase II livetime fraction in gray and exposure build-up in red. The previously published data is marked for [10] and [12].

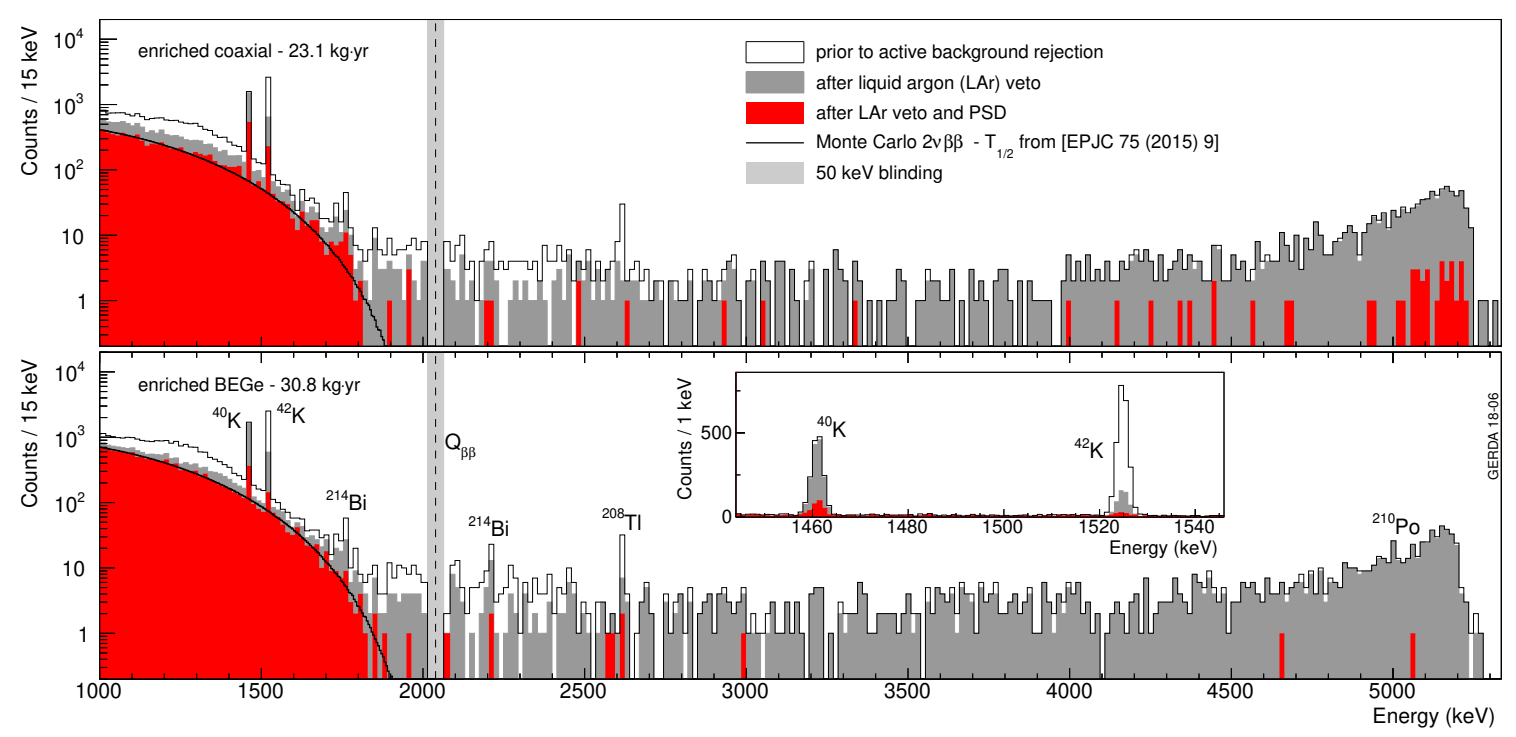

Figure 3: Energy spectra prior to unblinding. Consecutively the data is reduced by LAr veto (gray) and PSD cuts (red). The latter are only applied above $1 \mathrm{MeV}$. The blinded region $Q_{\beta \beta} \pm 25 \mathrm{keV}$ is marked in gray. For semi-coaxial detectors only data with improved alpha PSD is shown here. The inset in the lower panel displays the data reduction in the region of the ${ }^{40} \mathrm{~K}$ and ${ }^{42} \mathrm{~K}$ lines.

taking period are shown in Fig. 2. The accumulated data is blinded in a $\pm 25 \mathrm{keV}$ region around $Q_{\beta \beta}$, events falling in this energy window are unavailable for data analysis until unblinding. Two previously performed unblinding steps are marked [10, 12].

Weekly calibrations with a ${ }^{228} \mathrm{Th}$ sources ensure a stable energy scale in the whole data taking period. Periods in which this cannot be guaranteed are discarded from data analysis. A digital signal shaping filter with low-frequency noise rejection which is optimized for each calibration and each detector is used for energy reconstruction [13]. The full-width at half-maximum at $Q_{\beta \beta}$ is on average $(3.0 \pm 0.1) \mathrm{keV}$ for BEGe and $(3.6 \pm 0.1) \mathrm{keV}$ for semi-coaxial detectors.

The energy spectra obtained for semi-coaxial and BEGe detectors are shown in Fig. 3. Two lines are visible at $1460.8 \mathrm{keV}$ and $1524.6 \mathrm{keV}$ which are due to decays of ${ }^{40} \mathrm{~K}$ and ${ }^{42} \mathrm{~K}$. Except for these two components the spectra are dominated in the energy region below the ROI by the 


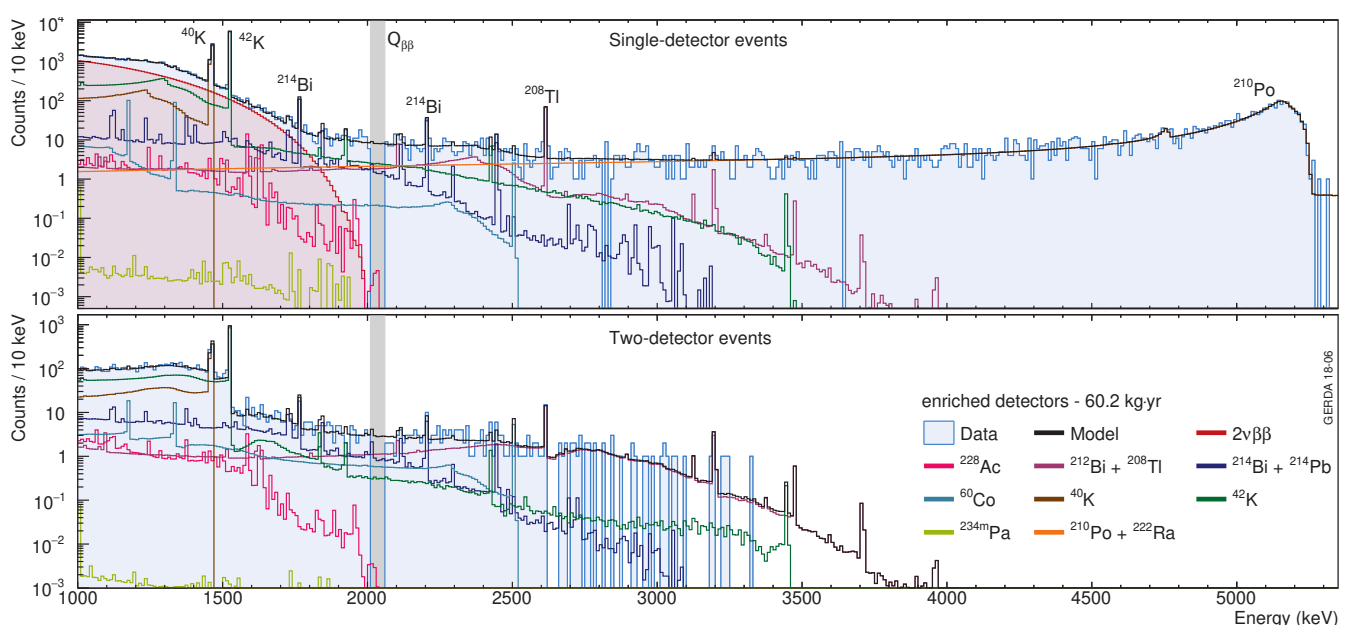

Figure 4: Different background components of the one- (top) and two-detector (bottom) sum energy spectra prior to LAr veto and PSD cuts. The blinded region of $\pm 25 \mathrm{keV}$ around $Q_{\beta \beta}$ is shaded in gray. The exposure shown is slightly higher than the $0 v \beta \beta$ analysis data set because the data selection is not as strict and also data which can not be used for PSD is kept here.

continuous spectrum of $2 v \beta \beta$ decay. The highest energy part of the spectrum is due to alpha decays - mostly of ${ }^{210} \mathrm{Po}$ - on the thin $\mathrm{p}^{+}$and groove surfaces of the detectors. Their observed energy is degraded due to the very short range of alpha particles.

Consecutive application of LAr veto and PSD are also highlighted in Fig. 3. The inset in the bottom panel shows the ${ }^{40} \mathrm{~K}$ and ${ }^{42} \mathrm{~K}$ lines. The LAr veto anti-coincidence cut is effectively reducing the ${ }^{42} \mathrm{~K}$ line due to simultaneous energy deposition due to the $\beta$ in the LAr. The ${ }^{40} \mathrm{~K}$ line is not affected as it originates from an electron capture process and no additional energy deposition is expected in this case. The signal acceptance of $0 v \beta \beta$ events due to the operation of the LAr veto is $(97.7 \pm 0.1) \%$.

For PSD of multiple-site events (MSE) in semi-coaxial detectors a neural network algorithm is used while for BEGe detectors this can be done with a mono-parametric method due to their particular internal field configuration [9]. The latter is based on the amplitude of the current pulse divided by the total integrated energy of a signal recorded with a BEGe detector. With a two sided cut MSE and surface alpha events are rejected. A new PSD approach developed to discriminate alpha events in semi-coaxial detectors based on the charge collection time allows to drastically reduce the background in this type of detector [14]. The new technique is applied only to data taken after the last unblinding in June 2017 and amounts to $23.1 \mathrm{~kg} \mathrm{yr}$ of exposure. The signal acceptance after PSD determined from $2 v \beta \beta$ events, calibrations and pulse shape simulations is $(71.2 \pm 4.3) \%$ for semi-coaxial detectors and $(87.6 \pm 2.5) \%$ for BEGe detectors in this data set.

The different background components prior to LAr veto and PSD are shown in Fig. 4. The decomposition is done via a Bayesian binned maximum likelihood fit based on material screening prior to their installation. In the top panel single detector events are displayed and in the bottom panel the sum energy of two detector events can be seen. Both spectra are fit simultaneously. The most important contributions in the ROI are due to the $\beta$ component of ${ }^{42} \mathrm{~K}$, alpha decays on the detector surface, ${ }^{208} \mathrm{Tl}$ from the thorium series and ${ }^{214} \mathrm{Bi}$ from the uranium decay chain. 


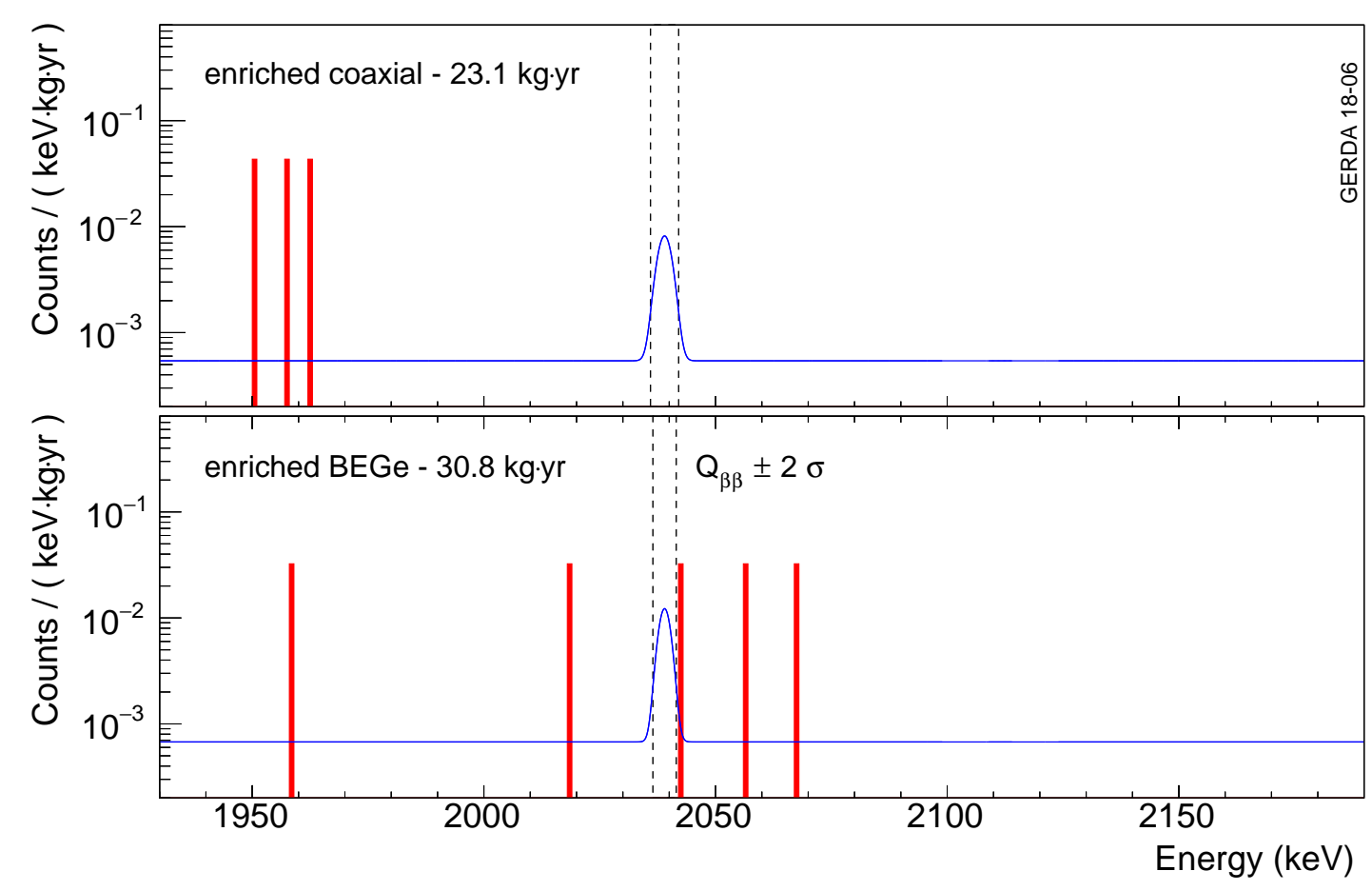

Figure 5: Unblinded energy spectra in the region around $Q_{\beta \beta}$ after LAr veto and PSD. In blue a hypothetical signal corresponding to the current half-life limit is shown.

The BI is determined in the region from $1930 \mathrm{keV}-2100 \mathrm{keV}$ excluding the ROI $( \pm 5 \mathrm{keV}$ around $Q_{\beta \beta}$ ), the single-escape peak from ${ }^{208} \mathrm{Tl}$ at $2103 \mathrm{keV}$ and a full energy peak at $2119 \mathrm{keV}$ originating from ${ }^{214} \mathrm{Bi}$. Fig. 5 shows this region for Phase II data after unblinding, LAr veto and PSD for semi-coaxial detectors after June 2017 and BEGe detectors. One event is observed in the BEGe detectors at an energy of $2042.1 \mathrm{keV}$ thus not accounted for in the determination of the BI. The energetic distance of this event to $Q_{\beta \beta}$ for the respective detector is $2.4 \sigma$. The determined BI based on this data set is very similar for semi-coaxial and BEGe detectors: $B I_{\text {coax }}=$ $\left(5.7_{-2.6}^{+4.1}\right) \cdot 10^{-4} \mathrm{cts} /(\mathrm{kg} \mathrm{keV} \mathrm{yr}), B I_{B E G e}=\left(5.6_{-2.4}^{+3.4}\right) \cdot 10^{-4} \mathrm{cts} /(\mathrm{kg} \mathrm{keV} \mathrm{yr})$.

The $0 v \beta \beta$ analysis is done via an unbinned maximum likelihood fit assuming a Gaussian signal over a flat background. All physics data collected in Phase I and Phase II are taken into account which amounts to a total exposure of $82.4 \mathrm{~kg} \mathrm{yr}$. This is $35.7 \mathrm{~kg} \mathrm{yr}$ in addition to the previous GERDA result [12]. The best fit is obtained assuming no signal. Thus a lower limit for the half-life of $0 v \beta \beta$ decay in ${ }^{76} \mathrm{Ge}$ is obtained. The frequentist analysis leads to a lower limit of $T_{1 / 2}^{\beta \beta}>0.9 \cdot 10^{26} \mathrm{yr}$ at $90 \%$ C.L. which is slightly weaker than the respective median sensitivity of $T_{1 / 2}^{\beta \beta}>1.1 \cdot 10^{26} \mathrm{yr}$ due to the event found at $2042.1 \mathrm{keV}$. The probability to observe one or more counts in $Q_{\beta \beta} \pm 3 \sigma$ given the observed background rate is $40.1 \%$ and the probability to obtain a stronger limit is $63 \%$. The frequentist half-life limit translates to an effective Majorana mass of $m_{\beta \beta}<110-250 \mathrm{meV}$ assuming a minimum of $g_{A} / N M A=1.25 / 2.81$ [15] and a maximum of $g_{A} / N M A=1.254 / 6.13$ [16]. In a Bayesian fit a half-life limit of $T_{1 / 2}^{\beta \beta}>0.8 \cdot 10^{26} \mathrm{yr}$ at $90 \%$ C.L. is obtained which is equal to the respective median sensitivity. 


\section{Conclusion and Outlook}

The very low background level in GERDA Phase II has been confirmed in the latest unblinding. A BI below the goal of $10^{-3} \mathrm{cts} /(\mathrm{keV} \mathrm{kg} \mathrm{yr})$ has been achieved not only with BEGe type detectors but also with the semi-coaxial detector geometry. Furthermore, the median sensitivity goal of $10^{26} \mathrm{yr}$ has been surpassed.

Currently the GERDA setup is being upgraded and additionally up to five newly produced inverted-coaxial detectors are being installed. Regarding the LAr veto a second fiber curtain close to the central detector string is added and the outer curtain is being replaced by a more densely packed one.

A first stage of the LEGEND experiment [17] has been confirmed by LNGS, in a first stage after 2019 the LEGEND Collaboration aims to operate $200 \mathrm{~kg}$ of enriched germanium detectors in the existing GERDA facility at LNGS and expertise from the MAJORANA [18] experiment even lower background levels are aimed to be achieved. Ultimately LEGEND wants to reach discovery sensitivity way beyond $10^{27} \mathrm{yr}$ and finally cover the entire parameter space of inverted neutrino mass hierarchy.

\section{References}

[1] Ackermann, K. H. and others, The GeRDA experiment for the search of $0 v \beta \beta$ decay in ${ }^{76}$ Ge, The European Physics Journal C 73 (2013) 2330.

[2] M. Agostini et al., Limits on uranium and thorium bulk content in GERDA Phase I detectors, Astroparticle Physics 91 (2017) 15.

[3] K. Freund et al., The performance of the Muon Veto of the GERDA experiment, The European Physical Journal C 76 (2016) 298.

[4] M. Agostini et al., Upgrade for Phase II of the GERDA experiment, The European Physical Journal C 78 (2018) 388.

[5] D. Budjás̆ et al., Isotopically modified ge detectors for GERDA: from production to operation, Journal of Instrumentation 8 (2013) P04018.

[6] M. Agostini et al., Production, characterization and operation of ${ }^{76}$ Ge enriched BEGe detectors in GERDA, The European Physics Journal C 75 (2015) 39.

[7] A. Lubashevskiy et al., Mitigation of ${ }^{42} \mathrm{Ar} /{ }^{42} \mathrm{~K}$ background for the GERDA Phase II experiment, The European Physical Journal C 78 (2018) 15.

[8] M. Agostini et al., LArGe: active background suppression using argon scintillation for the GERDA 0v $\beta \beta$-experiment, The European Physical Journal C 75 (2015) 506.

[9] M. Agostini et al., Pulse shape discrimination for GERDA Phase I data, The European Physical Journal C 73 (2013) 2583.

[10] M. Agostini et al., Background free search for neutrinoless double beta decay with GERDA Phase II, Nature 544 (2017) 47.

[11] M. Agostini et al., Results on Neutrinoless Double- $\beta$ Decay of ${ }^{76}$ Ge from Phase I of the GERDA Experiment, Physical Review Letters 111 (2013) 122503. 
[12] M. Agostini et al., Improved Limit on Neutrinoless Double- $\beta$ Decay of ${ }^{76}$ Ge from GERDA Phase II, Physical Review Letters 120 (2018) 132503.

[13] M. Agostini et al., Improvement of the energy resolution via an optimized digital signal processing in GERDA Phase I, The European Physical Journal C 75 (2015) 255.

[14] A. Zsigmond, New results from GERDA Phase II, Neutrino 2018 Conference.

[15] J. Menéndez et al., Disassembling the nuclear matrix elements of the neutrinoless $\beta \beta$ decay, Nuclear Physics A 818 (2009) 139.

[16] J. M. Yao et al., Systematic study of nuclear matrix elements in neutrinoless double- $\beta$ decay with a beyond-mean-field covariant density functional theory, Physical Review C 91 (2015) 024316.

[17] N. Abgrall et al., The large enriched germanium experiment for neutrinoless double beta decay (LEGEND), AIP Conference Proceedings 1894 (2017) 020027.

[18] N. Abgrall et al., The Majorana Demonstrator neutrinoless double-beta decay experiment, Advances in High Energy Physics 2014 (2014) . 